of our being bold in our declaration, let the consequences be what they may. The people who know nothing about it may make such declaration as they please, but it is the doctrine that fifty years hence will be the universal doctrine among scientific people.

Dr. Crothers-Mr. Chairman, I will just say one word with great thanks to the Chairman for his complimentary words to me, and his thorough recognition of the principles of my paper.

We are on the verge of a great reformation. In the courts all over the United States, we are all making the eternal blunder of sentencing diseased men, the same as they sentence the half insane and the witches and the possessed of the devil-we are doing the same thing in this country with the crazy inebriate. So the same blunder is repeated and we go on. Now regarding a man as insane or incompetent does not take away responsibility at all. Some people fear a great deal that to say because he is an insane man you should let him go loose. Not at all ; it only takes away the severity of the punishment. And I can only say that this is the view that is going to obtain in the coming century, and be put into practice; and the physician who adopts it as part of his life and his thought and in his practical application will first become the recognized man.

\section{REPORT OF TWO CASES OF INTRACRANIAL TUMOR.}

Read in the Section on Seurology and Medical Jurisprudence, at the Forty-tifth Annual Neetine of the American Medical tssociation, held at san Francisco, June o-s, 1894.

BY ANNE BURNET, M.D.

$$
\text { WAUSAL, Wis. }
$$

The following cases of intracranial tumor occurred in patients in the Illinois Eastern Hospital for the Insane.

The clinical history and post-mortem findings are given, not because of any marked peculiarity, so much as because of the growing interest in the subjectan interest which can but be stimulated and widened by reports of the careful observation of such cases.

It is a matter of regret that an. accurate diagnosis was not made before the patients reached the postmortem table, and there is a sort of selfish vindication in the thought that those of wide experience and having at their command the most approved means and methods of diagnosis sometimes fail to arrive at correct conclusions.

As knowledge of the brain and its functions increases improvements in methods of diagnosis may be looked for, until a tumor may be as accurately located in the skull as in the abdominal cavity.

Case 1.-Mary C., American, about 35̃ years of age. No history accompanied the patient when she was admitted to the hospital, and very little that could be relied upon was obtainable from her. She had lived a life of licentiousness, and the commitment papers gave alcohol as the cause of her mental disease. She was only fairly nourished. The pupillary reflex was normal; the tendon reflex absent. She walked with dificulty, her lower extremities seeming to be paretic. Her mouth was very foul-the gums swollen, spongy and exuding pus-presenting a good illustration of too much mercury. There was a profuse purulent discharge from the vagina and the patient said she had specific disease. Sensation seemed quite good at first but was soon evidently deranged. The patient's mental condition was such, however, that her statements in this regard as indeed in the matter of subjective symptoms in general could not be wholly relied upon. There was a slight hesitation in her speech and her memory was slow. She had hallucinations of sight and hearing accompanied by delusions of grandeur. These mental defects were not pronounced at first, but it is quite possible that change of surroundings may have thrown them into the background for a time as is often the case. Later, however, these mental symptoms were very marked and she became quite irritable.

She appeared to be weak and unable to get about and seemed to suffer so much from pain in all parts of her body that she was sent to the infirmary and permitted to remain in bed or to sit up as she preferred. She complained of very intense headaclse, claiming that she could not sleep on account of pain in her head and other parts of her body. The head pains were the only ones definitely located. The others seemed general and were suggestive of multiple neuritis. In a short time she became quite helpless and very untidy. She failed rapidly. Her stomach refused food and she suffered from an obstinate diarrhea. A few days before she died she developed a persistently high temperature and rapid pulse accompanied by copious perspiration. She was in the hospital less than six weeks. Before death for several hours the muscles of the neck became markedly rigid. She was quite deaf and there appeared to be a failure of sight also. The autopsy was made five and one-half hours after death. The head was small and the skull unusually thick. The diplöe were nearly obliterated. Exostoses were found on the inner surface of the frontal bone and in the temporal fossa on the left side. The brain weighed forty-one ounces. The dura was somewhat adherent to the skull and was thickened. The pia was thick, tough and unusually vascular. At a point corresponding to the exostosis in the left temporal fossa, the pia was roughened and granular to the touch. This was over the third temporal convolution and a point of red softening was found in this area. Signs of recent inflammation and a spot of red softening three millimeters. in diameter were found in the left frontal lobe. A large number of syphilomata were found in different parts of the brain as follows: A gummatous patch in the dura over the paracentral lobule on the left side; two tumors apparently developed from the pia over the occipital lobes causing corresponding depressions in those lobes; another at the top of the second frontal convolution extending into its substance at the expense of the gray matter; a large one at the upper border of the angular gyrus; one in the interparietal sulcus involving the gray matter on both sides of that fissure in the right hemisphere; one in the fissure of Rolando on the left side at about the middle of its extent; another at the tip of the cuneus in each hemisphere; one large and several small ones about the middle of the corpus callosum; one in first frontal sulcus on the right side anteriorly; one large one in the substance of the optic thalamus; several small ones buried in the surface of the cerebellum with others in the substance of its lobes; a large one in the pons showing as a projection in the floor of the fourth ventricle; second in the pons extending to the crura.

These tumors varied in size from a small pea to a goodsized hazel-nut and in most instances seemed to exist at the expense of the gray matter-appearing to rest directly upon the white substance of the brain. The white matter was in all parts unusually tenacious.

Some of the growths found in the sulci were rolled out on passing the finger tips between the convolutions. No similar growths were found in the lungs, liver, heart nor kidneys -but the spleen seemed to be literally filled with them.

The kidneys showed advanced fatty degeneration.

Considering the large number and wide distribution of these tumors it seems strange that there should have been so few marked symptoms. It is probable that the true physical condition was masked by her mental deterioration. The cerebral cortex was seriously pressed upon at several points, but there was nothing in the patient's condition until a few days before death to indicate such pressure. At no time after her admission did she have a convulsion. The persistent vomiting and reeling gait might have suggested cerebellar disease of some sort; and these symptoms together with the severe beadache complained of did suggest such a diagnosis. The patient's history, too, left little occasion to question the probable cause of the disease, although there had been no improvement under treatment.

The patient had evidently received a thorough course of mercury at least before she was sent to the hospital, judging from the condition of her month. The irritable condition of the stomach and bowels made all internal medication as well as the matter of nutrition very difficult.

It is stated in the books that while gumma occurs more frequently than any other form of intracranial tumor (except perhaps tubercular) it very rarely oceurs in the cerebellum. In this case several tumors were found in the cerebellum although much the larger number were in the cerebrum.

Case 2.-Mrs. O., Swede, 67 years of age, widow, mother of several children. No history pointing to organic brain dis- 
ease. The case appeared to be one of senile dementia. She had been well cared for, but a tendency to wander and very untidy habits made it necessary for her friends to place her in the hospital. On examination she was very well nourished; the reflexes were found to be fairly good, and no. disturbance of sensation was noted. Her sight and hearing seemed fair for her time of life. Her weakened mental condition made accurate information impossible. Her gait was her most noticeable defect. She walked very unsteadily with a rolling movement such as a sailor adopts in rough weather at sea. She was weak-minded, childish and affectionate - usually very cheerful, humming softly as she walked about or sat in the ward. She talked very little but seemed to think she expressed her sentiments in pleasant little sounds like a happy child.

One morning not long after her admission Mrs. O. was reported as having fainted at the breakfast table. On going to the ward she was found in bed where her attendant had placed her, looking very pale and as though she might be extremely nauseated. Her pulse was weak and slow, but steady, and she was inclined to sleep. She remained quietly in bed the greater part of that day and next morning was up and about as usual.

These attacks recurred a number of times, the patient seeming to be more helpless after each one. Her gait became more unsteady until she could not walk without assist- loss of sight and post-mortem findings confirmed that impression.

She died ten and one-half months after admission. The post-mortem was made thirteen hours after death. The body was well nourished. There was nothing unusual in the shape nor dimensions of the skull. The brain weighed forty-three ounces, and there was between five and six ounces of cerebro-spinal fluid. On the internal table of the calvarium in the frontal region were some fungosities. The caliber of the internal carotids at the point of entrance into the skull was greatly enlarged and the arteries at the base of the brain were also enlarged and atheromatous. The dura was very hyperemic and the walls of the longitudinal sinus were thickened. A bony nodule as large as a bean was found in the dura at a point corresponding to the upper end of the fissure of Rolando. On raising the frontal lobe to begin the removal of the brain a large tumor was discovered connected with and seeming to include the pituitary body-resting below on the sella turcica and pressing upward spreading apart all the basilar structures, carrying before it the infundibulum, the walls of which seemed to form a capsule for the growth. The tumor pressed against the anterior commissure anteriorly and greatly widened the optic commissure, against the pons and crura cerebri posteriorly and the anterior portion of the caudate nuclei laterlly. On removing the growth (which was nearly spherical

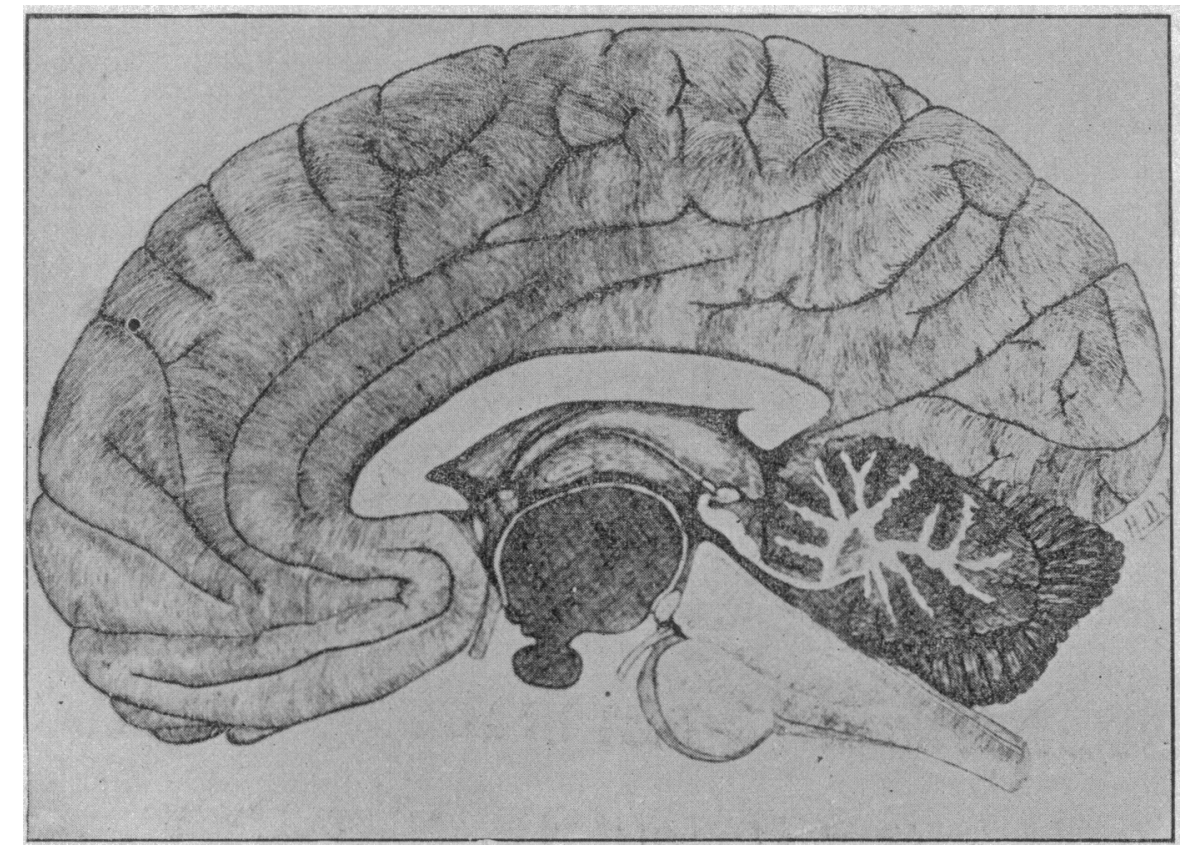

ance. Her mental faculties and powers of perception also failed. It was observed that she never seemed to notice a person unless directly in front of her, then when her attention was called she would smile pleasantly. This suggested the idea that the field of vision was limited but a satisfactory test was not made.

On account of her helpless condition and to prevent her from being injured by falling or by other patients, she was taken to the infirmary and put to bed. For a time she was very noisy and hard to care for. She was extremely untidy and required constant attention. The right leg soon began to contract at the knee and her general helplessness increased. When attempting to sit up in bed the muscles of the back seemed incapable of supporting the body, the tendency being for the body always to fall toward the left. She slept most of the time day and night. It was often necessary to arouse her to give her nourishment, etc. These changes were developed during about ten months. Her deafness increased until it was apparently complete and all other faculties seemed to fail in proportion. A short time before death she developed a temperature of 105 degrees on which baths and antipyretics failed to make any impression. At this time she began to keep her eyes closed continually, even when she took her food and medicine, which she did very well to the last. Her mental condition was such that it was not possible to discover the reason for keeping her eyes thus closed, but it was thought possibly to be due to and about twenty-eight millimeters in diameter) and its false capsule, large openings into the lateral ventricles were exposed. The third ventricle was greatly enlarged and mostly occupied by the neoplasm. The brain tissue in contact with the tumor, $i . e$. , the anterior half of the third temporal convolution, anterior portion of the caudate nuclei, the optic thalami and crura cerebri were softened from pressure necrosis. The island of Reil was apparently normal. The neoplasm was dark in color and its surface was irregular, almost bilateral. It was freely supplied with blood vessels by branches from the internal carotids.

The pia in all parts was intensely congested, especially at the bottom of the sulci and fissures. The line of demarcation between the white and gray matter was not clear in the portions of the brain near the tumor. The vessels in the floor of the lateral ventricles.were enlarged; the choroid plexus was apparently normal. Nothing unusual was noted in the fourth ventricle, and the cerebellum was apparently all right. On section the tumor was grayish in its interior and rather soft. By an oversight there was no microscopic examination made.

Dr. Valin made a drawing of the tumor in situ from which the accompanying photograph was taken. It is quite correct except that the pituitary body was inot so distinctly" separated from the neoplasm as the photograph would indicate.

In another case presenting symptoms similar to 
the one described, it was thought that the pituitary body might perhaps be abnormal. This patient died several months after the first, and on post-mortem examination the pituitary body was found to be noticeably enlarged and unusually firm. It could not be said to resemble the first, however.

There was a point of interest in this latter case which you will pardon my mentioning. Along the entire margin of the fold of pia which dipped down into the fissure of Rolando, on the left side, there was a succession of sago-like bodies of various sizes. This patient had several convulsions such as are not unusual in cases of paretic dementia, each attack leaving her more paretic until she was entirely helpless and bed-ridden.

I have found very little literature on tumors of the pituitary body. Gowers, in his latest edition, dismisses the subject in a short paragraph, while others merely mention the fact that tumors of that gland sometimes occur.

The tumor in the case described must have been of slow growth. No symptoms diagnostic of a new growth were noticeable until after the patient became bed-ridden. She never had a convulsion unless the attacks resembling a fainting spell could be so considered. Gowers speaks of a brief loss of consciousness resembling petit mal as occurring in some cases, and it is possible such may have been the nature of the attacks this patient had.

Headache and head pains are the most common symptoms of brain tumor, yet this patient never complained of either and never manifested any sign of suffering. She had attacks of vertigo and occasionally of vomiting, but her digestion was good and so was her appetite during her entire illness.

What the character of the growth was, I am sorry not to be able to state definitely. There was no history and no evidence of specific disease and no tubercular tendency. The tumor had not the appearance of a cancerous growth and there was no such growth elsewhere in the body.

Judging from its consistency, color and abundant blood supply and from the frequency of glioma, may it not have been a circumscribed glioma?

ON THE NATURE OF LOCOMOTOR ATAXIA.

Read in the Section on Neurology and Medical Jurisprudence at the Forty-fifth Annual Meeting of the American Medical Association, held at San Francisco, June 5-8, 1894.

BY L. HARRISON METTLER, A.M., M.D. MEDICAL EXAMINR NEDERLA ND LIFE INSURANCE COMPANY; CONSULTING

The intelligent treatment of a disease can only follow a thorough comprehension of its true nature. The management of locomotor ataxia has undergone many modifications until now it is beginning to be recognized that the very best results are obtained from a general constitutional treatment. Cauterizations, stretchings, and suspensions have all been tried and found wanting. Various specifics have been vaunted and quickly forgotten. Antisyphilitics continue still to benefit strictly syphilitic cases. At the present time, however, we rely chiefly on good food, change of scene, tonics, baths and properly regulated exercise. All this to me is very suggestive, and for the past few years I have been suspecting that the disease we call locomotor ataxia is but a manifestation of a general affection or cachexia. This cachexia is something more than a predisposing cause, and its cure means the cure of the nervous lesions and symptoms.

The etiology and pathology of locomotor ataxia, spastic paraplegia, ataxic paraplegia, amyotrophic lateral sclerosis, the disseminated, diffuse and even secondary scleroses are so similar while their differences are so insignificant that the grouping of them together, as some writers are doing, is entirely rational. When the same morbid process occurs in different parts of the brain, giving rise respectively to motor, sensory or special sense symptoms, we do not speak of them as different diseases. Neuralgia is the same disease in one nerve as it is in another, and the various special nerve palsies when dependent upon the same cause, are only distinguished by their differences of location. This is even more forcibly illustrated by considering together the three affections known as locomotor ataxia, hereditary ataxia and ataxic paraplegia, all of which bear a marvelously close relationship and are but different manifestations, as it were, of the same trouble. In regard to the lesion, Friedreich's disease stands as the connecting link between the other two. Its more limited extent in locomotor ataxia naturally defines more sharply the symptomatology of this disease; while in the more extensive form of the trouble, that known as ataxic paraplegia, the motor paralysis due to implication of the lateral tracts quite alters the ataxia dependent upon disease of the posterior columns. Every one knows that in hereditary ataxia there is more of an equality between the ataxia and paretic symptoms with a slight tendency, however, for the ataxia to predominate.

In all three diseases the congenital predisposition is more or less marked. It is probable that the hereditary influence is the same in all, but that sometimes, as in Friedreich's disease, it is so overwhelming as to institute a degeneration, or, perhaps more properly speaking, a prevention of development from the very beginning of embryonic life, while in the other two affections it remains milder or latent until awakened into activity by some special cause.

In accordance with the most recent physiology of the cord, we must materially alter our conceptions of the limitations of the lesion and the nature of the anesthesia that give rise to the ataxia of tabes dorsalis. The spinal tracts are far more complicated than we have hitherto been led to suppose. The posterior parts of the cord are not entirely sensory in function, nor are the anterior motor. Indeed, the most important centrifugal tract lies wholly behind a line drawn transversely through its center, while according to Gowers the chief centripetal fibers are to be found in front of this same line. Such able investigators as Lücke, Flechsig, and Bouchard have demonstrated the fact that in the posterior columns are systems of fibers endowed with most elaborate and complex functions. And as a realization almost of Reynolds' prophetic words, Charcot, Schultze and Strumpell have shown that the lesion of locomotor ataxia is not uniformly extended, but is most marked in particular sites and areas and that these same areas are affected with remarkable constancy and regularity. Westphal's discovery of the so-called pre-ataxic symptoms, most of which are to be attributed to the involvement of the reflex centers and functions of the cord, long ago intimated a wider extent and profounder state of disease than that of 